\title{
PAPER
}

TomoTherapy treatments of multiple brain lesions: an in-phantom accuracy evaluation

To cite this article: M Zani et al 2019 Phys. Med. Biol. 64025020

View the article online for updates and enhancements.

Workflaw

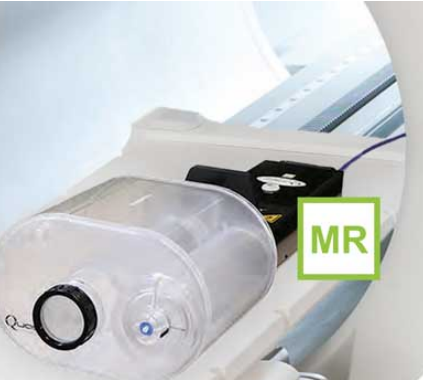




\title{
PAPER
}

\section{TomoTherapy treatments of multiple brain lesions: an in-phantom accuracy evaluation}

RECEIVED
24 July 2018

REVISED

26 November 2018

ACCEPTED FOR PUBLICATION

13 December 2018

PUBLISHED

18 January 2019

\author{
M Zani $^{1,4}$, L Marrazzo $^{2}$, S Calusi $^{1}$, C Talamonti ${ }^{1,2}$, S Scoccianti ${ }^{3}$, D Greto $^{3}$, I Desideri $^{1,3}$, F Fusi $^{1}$ and S Pallotta ${ }^{1,2} \odot$ \\ 1 Department of Biomedical, Experimental and Clinical Sciences 'Mario Serio', Viale Morgagni, 50, 50134 Firenze \\ Medical Physics Unit, Azienda Ospedaliero Universitaria Careggi, Largo Brambilla 3, 50134, Florence, Italy \\ 3 Radiotherapy Unit, Azienda Ospedaliero Universitaria Careggi, Largo Brambilla 3, 50134, Florence, Italy \\ 4 Author to whom any correspondence should be addressed. \\ E-mail:marghezani@gmail.com
}

Keywords: TomoTherapy, multiple brain metastasis, in phantom setup accuracy

\begin{abstract}
Aim of the present study was to evaluate the accuracy which can be obtained with helical TomoTherapy ${ }^{\circledR}$ (HT, Accuray) systems in the case of multiple intracranial targets treatments. Set-up accuracy was measured, for different registration options and MegaVoltage CT (MVCT) slice thickness, by applying known misalignments to an ad-hoc developed phantom. End-to-end (E2E) tests were performed to assess the delivery accuracy in phantoms containing multiple targets by using radiochromic films: measured dose distribution centroids were compared with physical and calculated target positions on axial and coronal planes. A Gamma index analysis was carried out on planned and measured planar dose maps. The bone and tissue algorithm with the fine MVCT reconstruction grid gave the best results among the automatic options. The most accurate registration modality resulted to be the manual one with a sub-voxel accuracy shifts and a capability in the detection of rotations within $0.3^{\circ}$. For the E2E along the coronal plane (six targets), a mean deviation between measured dose distribution centroids and physical barycenters of $0.6 \mathrm{~mm}$ (range $0.1 \mathrm{~mm}-1.3 \mathrm{~mm}$ ) was observed. Along the axial plane (five targets), a mean deviation of $1.2 \mathrm{~mm}$ (range $0.7 \mathrm{~mm}-2.1 \mathrm{~mm}$ ) was found for the centroids shifts. Gamma index $(5 \%, 1 \mathrm{~mm}$, local) passing rates higher than $87.5 \%$ between planned and delivered dose distributions were measured. These results demonstrate that multiple brain lesion HT treatments are feasible with an accuracy at least comparable to frameless linac-based delivery, when a set-up capable to assure angular corrections and a reliable patient immobilization is employed.
\end{abstract}

\section{Introduction}

Thanks to the advances in treatment planning and delivery, stereotactic radiotherapy has become a valuable clinical choice for the treatment of multiple brain metastases (Lee and Yamada 2017). In fact brain metastases can be efficiently treated with the only employment of highly conformed radiation beams concentrated to the lesion site or in combination with whole brain radiation therapy (WBRT) (Andrews et al 2004, Minniti et al 2011). Radiotherapy treatment with hypo fractionated schemes to small targets requires high accuracy in both lesion localization and dose delivery. The technical characteristics of Leksell Gamma Knife ${ }^{\circledR}$ (Schell et al 1995) and CyberKnife ${ }^{\circledR}$ (Dieterich et al 2011), covering both these aspects, make them elective modalities for that purpose. On the other side, due to the improvement of image guided delivery techniques, linac based protocols are also quite widespread (Wowra et al 2009, Treuer et al 2014, Cho et al 2015, Frakes et al 2015, Park et al 2016). In this framework the dose delivery method that characterizes helical TomoTherapy ${ }^{\circledR}$ (HT) (Langen et al 2010) systems could potentially provide an efficient and accurate alternative to the previously mentioned techniques. The continuous gantry rotation, combined with couch translation, and the non-isocentric approach could in fact lead to significant time saving when compared with other techniques: multiple lesions could be treated within a single delivery and therefore it would not require new patient set-up for each target. Nevertheless, only few 
works (Baisden et al 2007, Levegrün et al 2013, Nagai et al 2014, Barra et al 2015, Elson et al 2015) reported the employment of HT for the treatment of brain metastases.

Regarding the assessment of the HT treatment planning system (TPS) performances, some studies demonstrated that HT assures high performances in terms of both target coverage and organs at risk (OAR) sparing (Han et al 2006, Soisson et al 2006, Kumar et al 2010, Greto et al 2017). However, in order to perform stereotactic treatments, planning suitability is not sufficient, but reliable devices for patients immobilization and extremely accurate imaging systems for target localization are also mandatory.

These two critical aspects need to be investigated to evaluate whether a safe treatment of brain metastases can be guaranteed with HT. The present study focuses on the second critical point and was conceived as an end-toend (E2E) test to evaluate the accuracy which can be obtained with HT systems in the case of multiple targets. As a preliminary step, the set-up accuracy that HT imaging protocols and registration algorithms can provide was evaluated with an ad-hoc developed phantom. This is a prerequisite for the employment of HT with the objective of treating cranial lesions. Then, the E2E test was performed by evaluating the correspondence between the positions of multiple targets embedded in phantoms (both antrophomorphic and non) with planned and measured dose distributions.

\section{Materials and methods}

\subsection{HT set-up accuracy}

For this study a HT machine v.5.1.2 was employed. For HT systems, target localization is provided by registering MegaVoltage CT (MVCT) and CT images (Meeks et al 2005). Its accuracy depends on MVCT acquisition protocols and on image registration algorithms. In order to examine both these aspects, a phantom capable of mimicking different known misalignments was designed and developed and some specific MVCT acquisitions and registration tests were performed.

\subsubsection{MVCT acquisition protocols and image registration options}

Users can choose among three different MVCT slice thickness: fine $(2 \mathrm{~mm})$, normal ( $4 \mathrm{~mm})$ and coarse $(6 \mathrm{~mm})$, resulting in variable imaging durations (Yartsev et al 2007), image qualities and absorbed doses (Shah et al 2008, Mege et al 2016). The maximum acquisition length is $150 \mathrm{~cm}$ and the Field of View is $39 \mathrm{~cm}(512 \times 512$ pixels $)$.

The registration between planning CT and MVCT can be performed manually and by means of an automatic registration software which employs a modified version of the mutual information algorithm (Ruchala et al 2002) on the entire image. Moreover, 'Bone' and 'Bone and Tissue' options, where the registration algorithm uses only voxels with a density $>1.1 \mathrm{~g} \mathrm{~cm}^{-3}$ and between 0.3 and $1.1 \mathrm{~g} \mathrm{~cm}^{-3}$, respectively, are available. For time saving purposes, the image registration can be performed after images down-sampling, thus considering three resolution choices: superfine (SF, $512 \times 512$ pixels), fine (F, $256 \times 256$ pixels) and standard (S, $128 \times 128$ pixels).

The rotation components (yaw, roll and pitch) can be included in the registration process, but only the deviation around the longitudinal axis (roll) can be automatically adjusted by modifying the radiation source starting angle.

\subsubsection{Phantom description}

The phantom consists of a PMMA plate $1 \mathrm{~cm}$ thick with eight embedded glass spheres (GS-phantom) (figure 1(a)). On the PMMA plate two orthogonal lines were engraved for reference purposes and eight circular holes, in known positions with respect to the two orthogonal lines, were drilled using a numerical control milling cutter $(0.01 \mathrm{~mm}$ positioning accuracy). Eight glass spheres (GS) with a diameter of $1 \mathrm{~cm}$ and a density of $2.6 \mathrm{~g} / \mathrm{cc}$ (HUs for this material are 2900 for the CT and 1100 for the MVCT images) were embedded into the holes, thus simulating targets. The GS location was carefully chosen in order to allow an easy identification of each of them and, at the same time, to cover a wide range of spatial coordinates (reported in the table of figure 1(a)). The GSphantom can be positioned orthogonal (vertically) or parallel (horizontally) to the HT couch thus testing axial $(x-z)$ and coronal $(x-y)$ planes (figures $1(\mathrm{~b})$ and (c)).

\subsubsection{Measurements scheme}

The set-up accuracy was assessed by applying known misalignments to the GS-phantom and comparing them with the results of the registration between planning CT and MVCT.

In order to cope with the large number of possible combinations of MVCT acquisition protocols and image registration options, the validation procedure was carried out as a two steps process. In the first step, the most accurate registration algorithm was discovered by using the GS-phantom in the orthogonal configuration (figure 1(b)), while in the second step only the most accurate registration algorithm was used to complete the test with the GS-phantom in the parallel configuration (figure 1(c)). 
a)

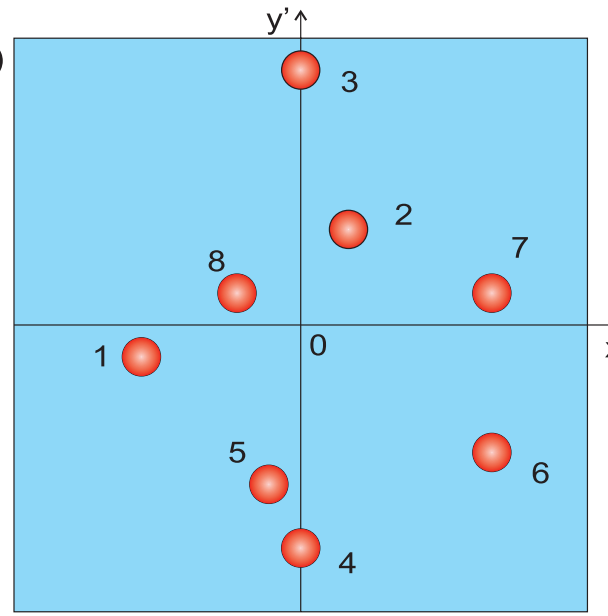

\begin{tabular}{|c|c|c|}
\hline & $x^{\prime}[\mathrm{mm}]$ & $\mathrm{y}^{\prime}[\mathrm{mm}]$ \\
\hline 1 & -50 & -10 \\
\hline 2 & 15 & 30 \\
\hline 3 & 0 & 80 \\
\hline 4 & 0 & -70 \\
\hline 5 & -10 & -50 \\
\hline 6 & 60 & -40 \\
\hline 7 & 60 & 10 \\
\hline 8 & -20 & 10 \\
\hline
\end{tabular}

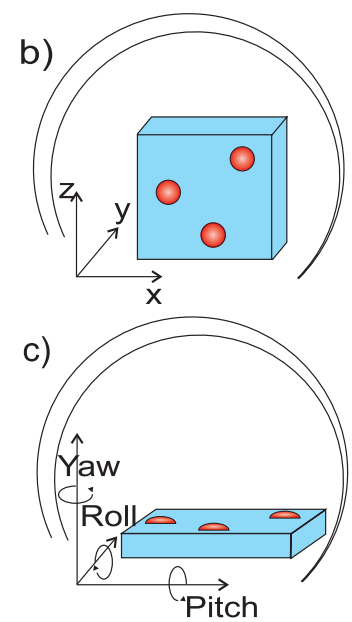

Figure 1. (a) Sketch of the GS-phantom, with the spheres position listed in the table. (b) Vertical orientation of the phantom on the HT couch with the HT coordinate system. (c) Horizontal orientation of the phantom with yaw, roll and pitch angles indicated.

\section{First step:}

A CT acquisition of the GS-phantom in the vertical configuration was performed with a Phillips Brilliance Big Bore, with a $512 \times 512$ matrix and $1 \mathrm{~mm}$ slice thickness $(0.75 \mathrm{~mm} \times 0.75 \mathrm{~mm}$ pixel size $)$ and sent to HT TPS (v5.0.2.6) where the usual in plane resolution degradation was applied, resulting in a pixel size of $1.5 \mathrm{~mm} \times 1.5 \mathrm{~mm}$. A treatment was planned (fine grid, voxel size of $1.5 \times 1.5 \times 1 \mathrm{~mm}^{3}$ ) aligning the red lasers with the two orthogonal lines engraved in the GS-phantom surface.

The GS-phantom was positioned on the HT couch and the localization lasers were deliberately aligned on a GS centroid rather than on the orthogonal reference lines, thus introducing known shifts of the GS-phantom in the $x$ - $z$ plane; this was repeated for each GS centroid. For each GS-phantom position, three MVCT studies were acquired (with fine, normal and coarse slice thicknesses) and registered on the planning CT data using manual, bone and bone and tissue options and the three possible image resolutions (SF, F and S). Differences between registration parameters and the applied shifts were evaluated. The registration accuracy was also assessed by calculating the residual error:

$$
R=\sqrt{\left(\Delta X^{\prime}-\Delta X\right)^{2}+\left(\Delta Y^{\prime}-\Delta Y\right)^{2}+\left(\Delta Z^{\prime}-\Delta Z\right)^{2}}
$$

where $\Delta X^{\prime}, \Delta Y^{\prime}$ and $\Delta Z^{\prime}$ are the obtained shifts registration parameters and $\Delta X, \Delta Y$ and $\Delta Z$ represent the known applied shifts.

For each position, a manual registration was also performed by one expert observer, as it is also carried out in the clinical practice. Regarding the inter-observer variability between CT and MVCT we have taken as representative of the present situation the repeatability assessed in the study of Boswell et al (2006) in which a mean standard deviation of $0.9 \mathrm{~mm}$ for 7 different registrations performed by 3 skilled observers was found.

\section{Second step:}

Shifts and rotations accuracy tests were completed using the GS-phantom in the horizontal configuration. A CT acquisition of the GS-phantom was performed (acquisition protocol previously described, resulting in a voxel size of $0.35 \times 0.35 \times 1 \mathrm{~mm}^{3}$ ) and sent to HT TPS where a treatment was planned (fine grid, voxel size of $\left.0.70 \times 0.70 \times 1 \mathrm{~mm}^{3}\right)$. As before, the GS-phantom was positioned on the HT couch and the localization lasers were aligned on each GS centroid rather than on the orthogonal reference lines, thus introducing known shifts of the GS-phantom in the $x$ - $y$ plan. For each GS-phantom position three MVCT studies were acquired using fine, normal and coarse slice thicknesses and registered on the planning CT data using the most accurate registration algorithm, as evaluated in the first step. The registration accuracy was again assessed evaluating the residual error. 
a)

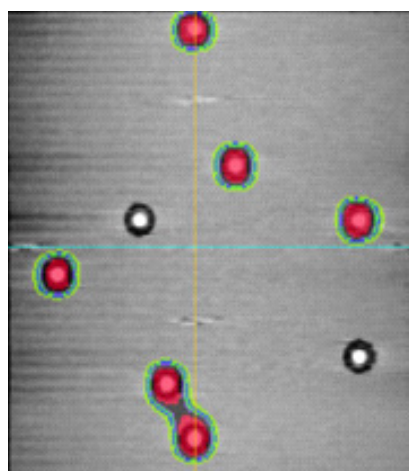

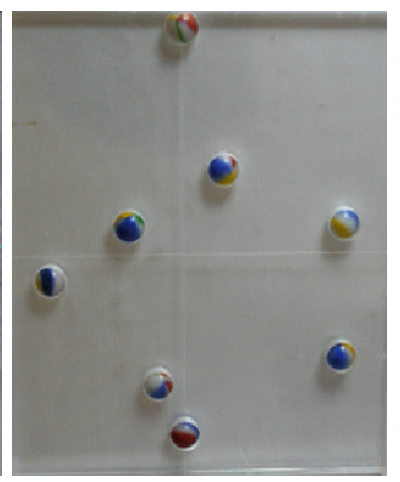

b)

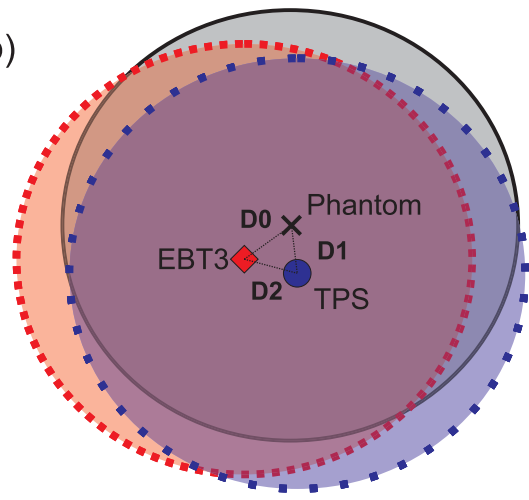

Figure 2. (a) Transverse planned dose distribution for the 6 lesions simulated on parallel GS phantom with a picture of the phantom itself. (b) Sketch of physical target superimposed with planned and EBT3 film measured dose distributions and centroids. In an ideal situation one should have D2 $=0$ and D0 = D1 and the dose distributions (both planned and measured) covering the entire physical target extension.

Finally, HT system capability to detect small rotations was tested. A support equipped with three screws and two digital levels permits to perform small rotations around $x$ (pitch) and $y$ (roll) axes (figure 1(c)). Rotations around $z$ axis (yaw) are measured with a precision goniometer using HT lasers and GSphantom orthogonal lines.

The GS-phantom was deliberately rotated of $0.5^{\circ}, 1^{\circ}$ and $2^{\circ}$ around $x, y$ and $z$ axes and MVCT images were acquired and registered on planning CT study using the most accurate combination of registration algorithm and acquisition protocol resulting from the previous tests. Differences between registration parameters and applied rotations were evaluated, together with residual errors.

\subsection{End-to-end test}

E2E tests were performed on phantoms and using GafChromic ${ }^{\mathrm{TM}}$ EBT3 (Ashland Inc. Wayne, NJ) films in order to assess the global delivery accuracy of the HT system. Also in this case measurements were performed on axial and coronal planes.

\section{Coronal plane:}

A plan was prepared simulating a treatment on six out of eight GS of the GS phantom (in figure 1(a) n. 1,2,3,4,5,7), delivering 20 Gy in 5 fractions to each target (figure 2(a)). A GafChromic EBT3 film was placed in correspondence of the GS, with the GS phantom in the parallel configuration. Prior to the delivery, the phantom position was accurately set by employing the most accurate registration technique and the best registration option, as assessed in the first part of the work. The test consisted in the evaluation of the correspondence between the physical position of the GS and the delivered doses distributions.

At first, for each target, the centroid position of the measured dose distribution was put in comparison with the physical position of the corresponding GS (i.e. D0, as sketched in figure 2(b), was measured). As a second step, also the shifts between TPS calculated dose centroids and physical ones (D1) were assessed. These shifts have to be taken into account as they represent the baseline shifts between dose distributions and the real target positions. Measured dose centroids locations were then compared also with TPS dose distributions, thus estimating D2 (figure 2(b)). In an ideal situation one should have D2 $=0$ and $\mathrm{D} 0=\mathrm{D} 1$.

GafChromic films that contained the informations on measured dose distributions, were for all cases digitized and carefully registered by means of FilmQAPro ${ }^{\mathrm{TM}}$ (Ashland Inc., Wayne, NJ and at www. filmqapro.com) software.

\section{Axial plane:}

In order to simulate multiple lesions inside an anthropomorphic phantom, three thin plastic sheets, were previously cut thus to create five approximately circular holes, and then they were inserted and fixed between adjacent slices of an Alderson Rando phantom head. In figure 3(a) a picture of the phantom with 
a)

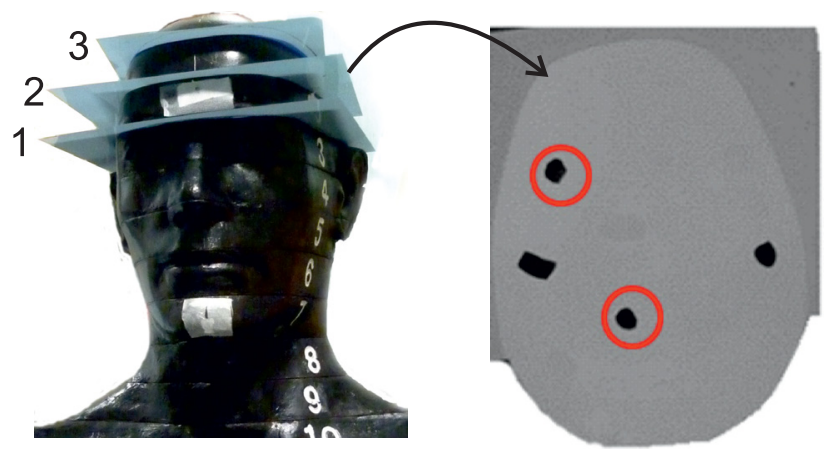

b)

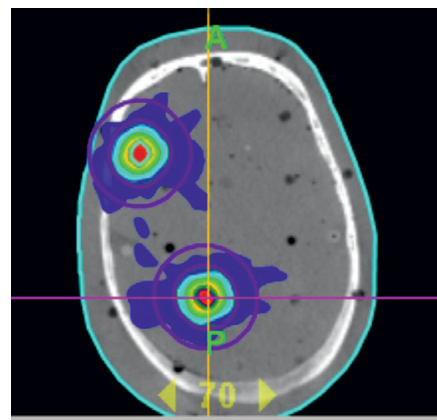

Figure 3. (a) Rando head Phantom with the three sheets inserted with the a picture of the central sheet. The red circles underline the two lesions contained in the sheet, while the other holes visible in the sheet are necessary for the positioning inside the phantom. (b) Axial view of planned dose distributions of the same two lesions.

the three sheets inserted is shown, together with the central sheet (n.2), containing two of the simulated lesions ( 5 targets in total, with the longest axis ranging from $4 \mathrm{~mm}$ to $8 \mathrm{~mm}$ ).

A CT acquisition $\left(0.67 \times 0.67 \times 2 \mathrm{~mm}^{3}\right)$ of the phantom was performed and sent to the HT treatment planning system. Each target was segmented and extended from the sheet to the three consecutive slices both in the cranial and caudal directions ( 7 slices in total for each target) thus reaching target volumes ranging from $0.47 \mathrm{cc}$ to $1.79 \mathrm{cc}$. A HT plan was prepared, delivering $20 \mathrm{~Gy}$ in 5 fractions to each lesion (figure 3(b)). A GafChromic EBT3 film was fixed on each plastic sheet, and sandwiched in between the Rando slices. The phantom, with GafChromic EBT3 films fixed on each plastic sheet, was positioned on the HT couch by employing the same support previously used in the rotations test. The phantom position was again verified with the most accurate registration technique. After the delivery, a careful registration between EBT3 films and both CT plastic sheets and TPS planar dose distributions was carried out. A centroids location analysis aiming to the evaluation of D0, D1 and D2 (figure 2(b)) was thus performed also along the axial plane.

Planned and measured dose maps were compared by means of FilmQAPro ${ }^{\mathrm{TM}}$ following, for the calibration and handling of EBT3 films, the protocol described in Marrazzo et al (2015). In order to compare these dose maps in terms of absolute values a procedure was followed that consisted of checking the output of the machine with a reference ion chamber (Extradin A1SL ion chamber (standard imaging)) and of rescaling dose values measured by EBT3 films by performing measurements, prior to the acquisition of the maps, with both EBT3 and ion chamber placed in the Cheese Phantom (Gammex Inc., Middleton, WI), a cylindrical solid water phantom supplied by TomoTherapy, Inc.

\section{Results}

\subsection{HT set-up accuracy}

\section{First step:}

In figure 4 the results of the first test, performed to evaluate the best registration option (manual, bone and bone and tissue) are reported. For each combination of acquisition protocol and registration algorithm the mean $\mathrm{R}$ value and standard deviation over the 8 tested targets were evaluated and plotted. Lower $\mathrm{R}$ values are generally obtained for the bone and tissue algorithm, when they are compared with the Bone algorithm. For each set of data, no significant differences are instead noticeable between the three reconstruction resolutions ( $\mathrm{S}, \mathrm{F}, \mathrm{SF}$ ), while some distances in the $\mathrm{R}$ values can be seen when the MVCT resolution changes, with a lower residual error measured for fine MVCT slice thickness. The best results in terms of $\mathrm{R}$ value among the automatic modalities are thus obtained when the bone and tissue algorithm with the fine resolution MVCT grid is used.

For the Bone algorithm a high variability of data is found, with registered shifts that in some case are clearly wrong (a vertical shift higher than $10 \mathrm{~mm}$ has been recorded).

The most accurate registration modality results to be the manual one ( $M$, in figure 4 ) with mean $R$ values of $1.3 \pm 0.6 \mathrm{~mm}, 1.1 \pm 0.6 \mathrm{~mm}$ and $1.3 \pm 0.1 \mathrm{~mm}$ for the fine, normal and coarse MVCT acquisition mode, respectively.

In all cases rotations were monitored and resulted to be inside $0.3^{\circ}$. 


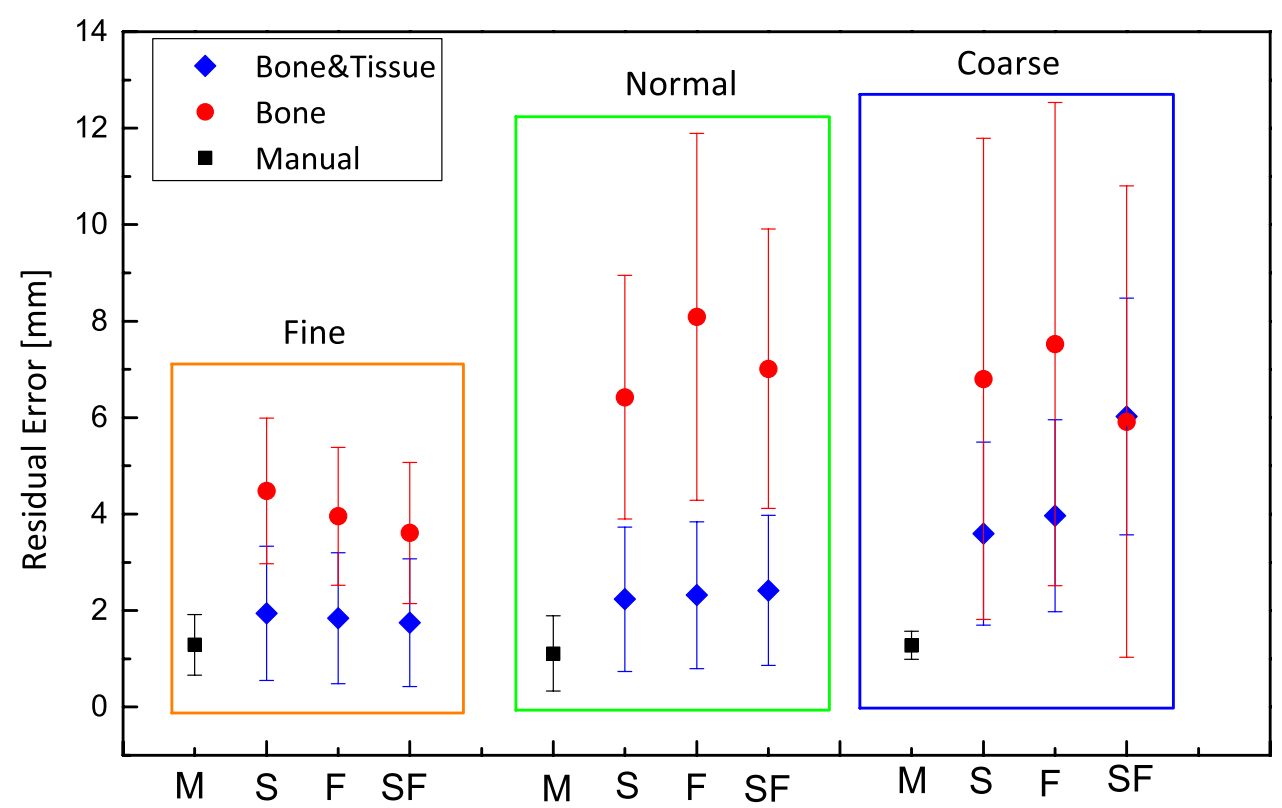

Figure 4. Mean R values of the results for automatic Bone (red), bone and tissue (blue) algorithms and manual (M, black) registrations as a function of the image resolution employed (standard (S), fine (F) and superfine (SF)). In the red rectangle the results when the MVCT is carried out with fine slice thickness, in green the normal and in blue the coarse. The error bar is of 1 SD.

\section{Second step:}

In table 1 residual $R$ values between applied and measured registration parameters obtained using the manual registration algorithm and with the phantom in the horizontal configuration are reported.

When the MVCT fine resolution is used, there are slightly better results, as reflected by a lower mean $\mathrm{R}$ value $(0.9 \mathrm{~mm} \pm 0.3 \mathrm{~mm})$. In this case, absolute mean differences between applied shifts and known values were $0.3 \pm$ $0.2 \mathrm{~mm}, 0.5 \pm 0.5 \mathrm{~mm}$, respectively along lateral $(x)$ and longitudinal $(y)$ directions.

In all performed tests, possible rotations were monitored and resulted to be negligible, as well as vertical translations. The results of the system accuracy in detecting deliberately applied rotations along the three axes (tested using MVCT fine resolution and manual registration algorithm) are illustrated in table 2, that reports the registration parameters obtained for all applied rotations and the set angles. Data show that phantom inclination can each time be detected correctly by performing a fine MVCT, with an accuracy, for the angles range investigated, of $0.3^{\circ}$, evaluated as the maximum difference between set and measured angles. In all performed tests, shifts resulted to be within the set-up procedure uncertainty $(0.2 \mathrm{~mm})$ which was determined by repeating the phantom set-up ten times and evaluating the maximum differences among registration parameters.

\subsection{End-to-end test}

\section{Coronal plane:}

In figure 5(a) the $x$ - $y$ plane GS centroid physical positions are shown together with EBT3 measured and TPS calculated dose distributions centroids. In figure 5(b) the measured shifts (D0, D1 and D2) are reported. D0 values are within $1.33 \mathrm{~mm}$, representing the very good correspondence between delivered dose distributions and physical targets. Regarding the dose distributions comparisons (EBT3 films measured versus TPS calculations) the worst situation is found for sphere 3 , for which a maximum D2 of $1.5 \mathrm{~mm}$ has been measured. Globally, the measured shifts are all within the pixel size dimension $(0.70 \mathrm{~mm}$ $\times 1 \mathrm{~mm}$ ), with mean values for D0, D1 and D2 inside $1 \mathrm{~mm}$.

\section{Axial plane:}

Results of the registration between plastic foils, EBT3 measured and TPS calculated dose maps are reported in figure 6. Qualitative results of the registration are reported in figure 6(a) for two of the five targets (Slice1). In figure 6(b), planned and measured orthogonal dose profiles relative to one of the two lesions are reported. From the two figures it is possible to observe that measured dose distributions perfectly correspond and almost overlap to both physical targets and TPS dose distributions; analogous results are found for the other analyzed lesions. 
Table 1. Manual registration: residual R value for the differences between the registration results and the deliberately applied shifts along $x$ and $y$ axes.

\begin{tabular}{llll}
\hline & Fine & Normal & Coarse \\
\hline Position & $\mathrm{R}(\mathrm{mm})$ & $\mathrm{R}(\mathrm{mm})$ & $\mathrm{R}(\mathrm{mm})$ \\
\hline 1 & 0.5 & 0.7 & 0.4 \\
2 & 1.2 & 1.2 & 0.3 \\
3 & 1.2 & 0.8 & 1.2 \\
4 & 0.7 & 1.4 & 1.7 \\
5 & 0.6 & 1.1 & 1.1 \\
6 & 0.9 & 1.3 & 1.2 \\
7 & 1.1 & 1.1 & 2.0 \\
8 & 1.3 & 1.7 & 2.2 \\
Mean & $\mathbf{0 . 9}$ & $\mathbf{1 . 2}$ & $\mathbf{1 . 3}$ \\
St. Dev. & $\mathbf{0 . 3}$ & $\mathbf{0 . 3}$ & $\mathbf{0 . 7}$
\end{tabular}

Table 2. Angles obtained by the registration put in comparison with set up rotations.

\begin{tabular}{llll}
\hline Set angle & Registered rotations & \\
\hline Yaw $\left(^{\circ}\right)$ & Pitch $\left(^{\circ}\right)$ & Roll $\left(^{\circ}\right)$ & Yaw $\left(^{\circ}\right)$ \\
\hline-0.5 & 0 & -0.03 & -0.6 \\
-1 & 0 & -0.2 & -1.0 \\
-2 & 0 & -0.03 & -2.0 \\
\hline Pitch $\left(^{\circ}\right)$ & Pitch $\left(^{\circ}\right)$ & Roll $\left(^{\circ}\right)$ & 0 \\
0.5 & 0.5 & -0.1 & 0 \\
1 & 1.1 & -0.03 & 0 \\
\hline 2 & 2.0 & -0.1 & 0 \\
\hline Yoll $\left(^{\circ}\right)$ & Pitch $\left(^{\circ}\right)$ & Roll $\left(^{\circ}\right)$ & Yaw $\left(^{\circ}\right)$ \\
\hline 0.5 & 0 & 0.5 & 0 \\
1 & 0 & 1.0 & 0.3 \\
2 & 0 & 2.0 & 0.2
\end{tabular}

The table in figure 6(c) reports centroids shifts, thus showing the quantitative analysis results. Mean values for D0 and D2 are within the pixel dimensions $(1.3 \mathrm{~mm} \times 1.3 \mathrm{~mm})$ along the axial plane. The maximum global difference between physical centroids and the EBT3 film measured dose distributions is found for sheet 3 , where a D1 of $2.16 \mathrm{~mm}$ has been found. For this target a distance of $1.73 \mathrm{~mm}$ has also been measured for the shift between the planned centroid position and the physical one, while a good correspondence between measured and planned positions has been found (D2 $=0.22 \mathrm{~mm}$ ).

A quantitative evaluation of the agreement between measurements and TPS calculations regarding dose distribution in their whole was obtained by performing a Gamma analysis on each EBT3 sheet. The criteria employed to compare the measured and calculated maps were $5 \% / 1 \mathrm{~mm}$ and $2 \% / 2 \mathrm{~mm}$ (local approach). Results are shown in table 3. Even after a optimization of the Gamma passing rates, which is obtained by shifting and rotating one map respect to the other, only a slight increase of the results can be achieved, but with minimal shifts and rotations respect to the maps aligned thanks to the fiducials.

\section{Discussion}

Nowadays many centers employ linacs to perform hypo-fractionated treatments on brain metastases, but some doubts still exist on the HT capability of assuring the same performances and an adequate accuracy. On this subject, a really interesting work was presented by Soisson et al (2010) in which procedures for testing the delivery accuracy of the TomoTherapy system were described, for cases similar to the ones presented in this work. The accuracy of the delivery was found to be within \pm 1 voxel dimension. These results are well within conventional stereotactic treatments tolerances and compare favorably to other linear accelerator based techniques. However, the one described is a specific radiosurgery workflow: the planning is performed on the MVCT images, with a single day schedule and with the patient immobilized during the whole procedure, so a specific setup is used.

Our study aimed to give some general results and indications on feasibility of stereotactic HT treatments regarding the set-up and global delivery accuracy issues, i.e. not directly connected to a particular workflow, 


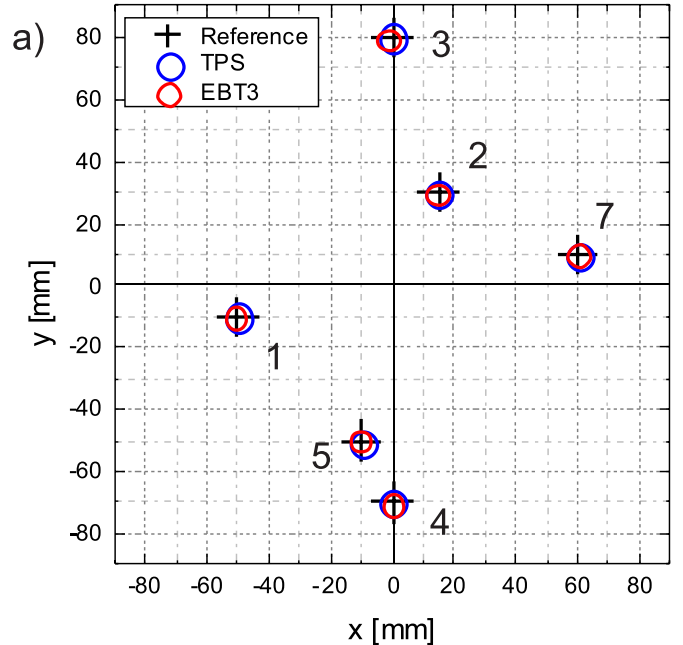

b)

\begin{tabular}{|c|c|c|c|}
\hline & $\begin{array}{c}\text { D0 (EBT3-Ph) } \\
{[\mathrm{mm}]}\end{array}$ & $\begin{array}{c}\text { D1 (TPS-Ph) } \\
{[\mathrm{mm}]}\end{array}$ & $\begin{array}{c}\text { D2 (EBT3-TPS) } \\
{[\mathrm{mm}]}\end{array}$ \\
\hline $\mathbf{1}$ & 0.63 & 0.73 & 1.03 \\
\hline $\mathbf{2}$ & 0.77 & 0.65 & 0.62 \\
\hline $\mathbf{3}$ & 1.33 & 0.37 & 1.52 \\
\hline $\mathbf{4}$ & 0.45 & 0.39 & 0.08 \\
\hline $\mathbf{5}$ & 0.36 & 0.89 & 1.15 \\
\hline $\mathbf{7}$ & 0.11 & 0.68 & 0.63 \\
\hline Mean & $\mathbf{0 . 6 1}$ & $\mathbf{0 . 6 2}$ & $\mathbf{0 . 8 4}$ \\
\hline St. Dev. & $\mathbf{0 . 4 2}$ & $\mathbf{0 . 2 0}$ & $\mathbf{0 . 5 0}$ \\
\hline
\end{tabular}

Figure 5. (a) Locations of theoretical (reference), EBT3 measured and TPS calculated GS centroids, for the five simulated GS targets, in the $x-y$ plane. (b) Table reporting the distances between physical centroids, EBT3 measured and TPS calculated dose distributions barycenters for each GS analyzed, together with mean value and standard deviation.

a)
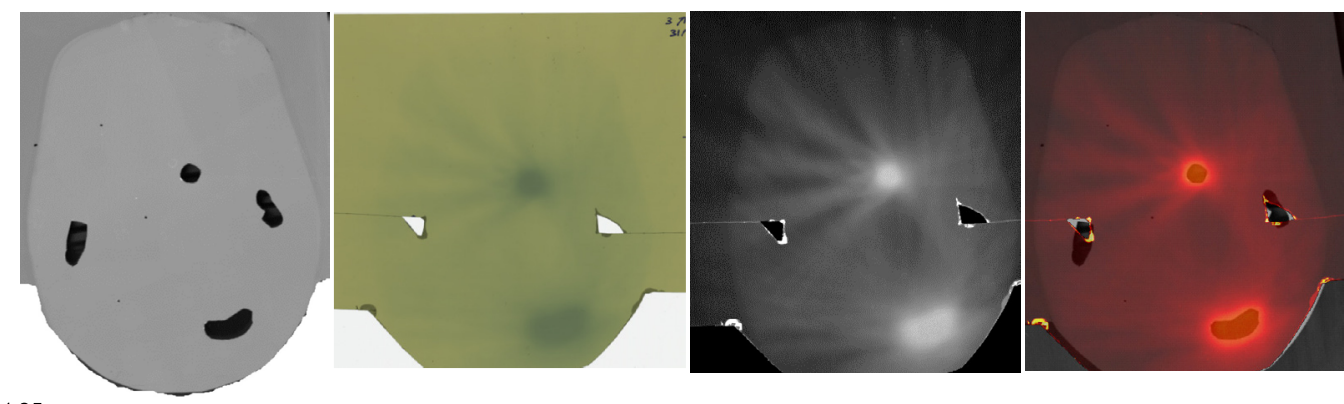

b)

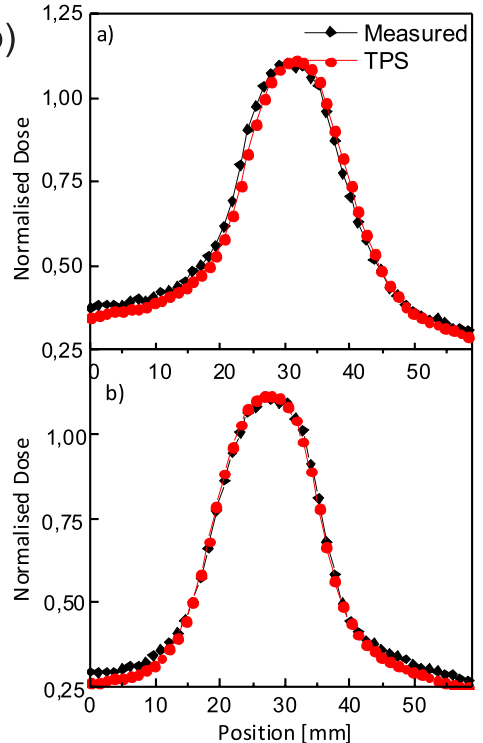

c)

\begin{tabular}{|c|c|c|c|}
\hline & $\begin{array}{c}\text { D0(EBT3-Ph) } \\
{[\mathrm{mm}]}\end{array}$ & $\begin{array}{c}\text { D1(TPS-Ph) } \\
\text { [mm] }\end{array}$ & $\begin{array}{c}\text { D2(EBT3-TPS) } \\
\text { [mm] }\end{array}$ \\
\hline Slice1 Target1 & 1.53 & 1.34 & 0.80 \\
\hline Slice1 Target2 & 0.82 & 1.18 & 0.51 \\
\hline Slice2 Target1 & 0.71 & 1.73 & 1.32 \\
\hline Slice2 Target2 & 0.79 & 1.26 & 0.67 \\
\hline Slice3 Target1 & 2.16 & 1.73 & 0.22 \\
\hline Mean & 1.20 & 1.45 & 0.70 \\
\hline St. Dev. & 0.63 & 0.26 & 0.41 \\
\hline
\end{tabular}

Figure 6. (a) from left to right: for the most caudal slice (n.1): the plastic sheet, the irradiated EBT3 film, the EBT3 film converted in dose, and the superposition between the physical sheet and the measured dose distribution. (b) Horizontal and vertical dose profiles of one of the two lesions of sheet $n .1$ as results of the fiducials alignment between measured and calculated dose maps. In red the TPS planned dose is reported, in black the EBT3 measured profile is shown. (c) Table reporting the distances between physical centroids, EBT3 measured and TPS calculated dose distributions barycenters for each target, together with mean value and standard deviation. 
Table 3. Second column: Gamma passing rates obtained from the fiducials registration between EBT3 films and TPS dose distributions for the three sheets inserted in the Rando head phantom. Third-sixt columns: optimized Gamma passing rates together with the shifts and rotations needed to switch from the original registration to the optimized one. For each film both the $5 \% / 1 \mathrm{~mm}$ and $2 \% / 2 \mathrm{~mm}(\mathrm{local}$ approach) criteria were employed and reported.

\begin{tabular}{|c|c|c|c|c|c|}
\hline & Gamma 5\%1 mm & & Shifts & & \\
\hline & Fiducials (\%) & Optimized (\%) & $x(\mathrm{~mm})$ & $y(\mathrm{~mm})$ & Rotation $\left({ }^{\circ}\right)$ \\
\hline Film1 & 91.3 & 93.6 & 0.1 & 0.2 & 0.3 \\
\hline Film2 & 95.0 & 95.3 & 0.3 & 0.3 & 0.3 \\
\hline \multirow[t]{3}{*}{ Film3 } & 87.5 & 92.2 & 0.5 & 0.2 & 0.9 \\
\hline & Gamma $2 \% 2 \mathrm{~mm}$ & & Shifts & & \\
\hline & Fiducials (\%) & Optimized (\%) & $x(\mathrm{~mm})$ & $y(\mathrm{~mm})$ & Rotation $\left({ }^{\circ}\right)$ \\
\hline Film1 & 91.3 & 91.7 & 0.5 & 0.1 & 0.4 \\
\hline Film2 & 88.2 & 89.6 & 0.2 & 0.4 & 0.5 \\
\hline Film3 & 88.6 & 89.5 & 0.5 & 0.6 & 0.7 \\
\hline
\end{tabular}

support, or immobilization system. Two phantoms were used in order to test both set-up and delivery in three dimensions, taking also into account possible rotations.

First of all, MVCT performances were tested, in order to understand if this imaging technique could be used for stereotactic localization. Registrations along the $x-z$ and $x-y$ planes, in the case of known applied shifts and rotations, were performed with the employment of a simple in house phantom. The results obtained are in agreement with what can be found in the literature about the MVCT performances (Boswell et al 2005, Mahan et al 2005, Boswell et al 2006, Woodford et al 2007, Vaandering et al 2009), indicating a very good sensibility and accuracy of the order of voxel dimension, that is the factor that should determine the ultimate precision limit. For our set-up, the manual registration resulted to be the most accurate modality whether, among the different automatic techniques, the bone and tissue algorithm gave the best results. The results found for the Bone algorithm are in some cases clearly wrong, with registered shifts higher than $10 \mathrm{~mm}$. This is probably due to the combination of the algorithm used with the GS-phantom high density inserts shape and position: the regularity in the placement of the GS and the fact that they are identical may cause the malfunctioning of the algorithm.

As demonstrated by Boswell et al (2006) the time to compute an automatic registration is considerably shorter than the time required for manual registration. If one aims at increasing the registration speed, with our setup, an option could be thus to perform an automatic registration by using the bone and tissue followed by a manual check.

A R value slightly lower $(0.9 \mathrm{~mm}$ versus $1.3 \mathrm{~mm})$ was found for the parallel set-up, comparing the manual registration results for the two orientations, and this is a consequence of the smaller CT voxels dimension in the transverse $(x-z)$ plane $\left(0.35 \times 0.35 \mathrm{~mm}^{2}\right.$ versus $\left.0.75 \times 0.75 \mathrm{~mm}^{2}\right)$. On the contrary, along $y$ axis, no difference is noticeable between the two orientations (mean shifts equal within SD), and this reflects the fact that the CT slice thickness is the same $(1 \mathrm{~mm})$.

Furthermore we showed that small rotations are detectable with a precision within $0.3^{\circ}$ and, as far as we know this is the first time that a similar check has been performed.

An accurate rotational detection and correction is necessary in order to perform stereotactic treatments. For this reason, in order to treat a real patient, a setup capable of rotational correction has to be employed, since it is not conceivable that the correct position is sought by changing the patient position with a trial and error process. Nowadays it is possible to find head supports that are capable of pitch corrections such as Uni-frame ${ }^{\circledR}$ Tilting Baseplate (CIVCO Radiotherapy) and couch extensions such as FreedomX ${ }^{\mathrm{TM}}$ Couch Extension (CDR systems) that correct for both pitch and yaw. The last device is at the moment unfortunately not compatible with the TomoTherapy couch. On the other side some papers try to predict how much the uncorrected rotational corrections translate to dose accuracy and to perform a tolerance evaluation, in the case of stereotactic brain treatments. Gevaert et al (2012) compared the calculated dose with and without rotational corrections measuring a loss of $5 \%$ dose coverage when rotational correction was not used, and pointing to a $0.5^{\circ}$ rotation error as a threshold angle for correction. Boman et al (2016) found that, for typical rotation errors seen in clinical practice, a treatment plan of comparable quality to $6 \mathrm{D}$ rotation correction could be achieved by using $6 \mathrm{D}$ registration without a rotational correction in the selection of patient positioning. This procedure assured, for a quadratic sum of rotation values of $2^{\circ}-3^{\circ}$, still adequate plan quality values.

Our study was conceived as an end-to-end test for the treatment of multiple lesions with HT. The analysis performed on the centroid shifts demonstrates the very good conformity of the delivered dose distribution to the targets. Centroids shifts in the coronal plane were found within $1.5 \mathrm{~mm}$ for all cases. For the axial plane, a mean value of $1.2 \mathrm{~mm}$ (range $0.7 \mathrm{~mm}-2.1 \mathrm{~mm}$ ) for the shifts between EBT3 film measured and physical barycenters 
was found and a mean value of $0.7 \mathrm{~mm}$ (range $0.2 \mathrm{~mm}-1.3 \mathrm{~mm}$ ) was obtained between measured and planned dose centroids. Moreover, for the simulated lesions inside the Rando head, planned and measured dose maps were compared, as in a real patient pre-treatment verification. The local Gamma passing rates obtained for EBT3 measured maps were very good and are in agreement with what can be found in the literature. These results are within the tolerances requested for stereotactic treatments (Langen et al 2010).

The HT delivery method is characterized by a continuous gantry rotation combined with couch translation, with a non-isocentric approach and a pneumatically driven collimator. The leaf size is $0.625 \mathrm{~cm}$, the smaller collimation (field width) in cranio-caudal direction is $1.05 \mathrm{~cm}$. Due to the peculiarity of the delivery, HT treatment plan quality has to be addressed in order to assess the reliability of TomoTherapy treatments on small brain targets. While the present study was conceived to explore the accuracy, mainly geometrical, that can be reached in HT, its planning performances are the focus of some papers (Han et al 2006, Soisson et al 2006, Kumar et al 2010, Greto et al 2017) in which parameters such as PTV coverage and conformity and OAR doses are evaluated and compared with other delivery techniques. In the work of Han et al (2006) the authors compare TomoTherapy with non coplanar IMRT ( $5 \mathrm{~mm}$ leaf-width Linac) for brain targets and they conclude that better dose conformity and dose gradient are reached in TomoTherapy plans compared with step- and-shoot IMRT plans for intracranial stereotactic radiosurgery. Greto et al (2017) investigated the HT capabilities in the planning of brain metastasis. They concluded that CyberKnife and HT are both optimal SRS devices, the choice to use one over another has to be clinically guided. In the light of this, for the treatment of very small brain metastasis, of comparable dimension respect to the TomoTherapy leaf width $(6.25 \mathrm{~mm}$ along the $x$-direction), a careful evaluation of the dosimetric parameters of the plan to be delivered (such as PTV coverage, conformity index, OAR maximum and mean doses) has to be performed thus to assess if it could represent an efficient clinical choice. A pre-treatment single patient verification is also mandatory.

Moreover, even if in our study the possibility of safely using HT for treatment of multiple brain metastases was demonstrated by mimicking the realistic situation of a treatment with a non-invasive fixation system, it is always important to keep in mind that, in a real patient treatment, contributions to the uncertainty in dose delivery are also due to other sources such as patient intra-fraction motions (Ramakrishna et al 2010, Floriano et al 2013, Bichay and Mayville 2016).

\section{Conclusions}

The set-up precision of the HT MVCT was investigated on an in-house phantom: a procedure to follow and a series of parameters that can assure sub-voxel accuracy for shifts and a high precision detection of rotations was found.

End-to-end tests showed the accuracy of the HT system in the delivery of multiple lesion treatments, represented by a very good conformity between planned and measured dose maps.

These results demonstrate that, with a set-up capable to assure angular corrections and a reliable patient immobilization, multiple brain lesion HT treatments are feasible with an accuracy that is at least comparable to frameless linac-based delivery.

\section{Acknowledgments}

This work was funded thanks to the support of the Soroptimist Club of Florence, Italy-Award in memory of Prof Raffaella De Dominicis for young women involved in STEM.

\section{ORCIDiDs}

S Pallotta (10 https://orcid.org/0000-0003-4123-7971

\section{References}

Andrews D W et al 2004 Whole brain radiation therapy with or without stereotactic radiosurgery boost for patients with one to three brain metastases: phase iii results of the rtog 9508 randomised trial Lancet 363 1665-72

Baisden J M, Benedict S H, Sheng K, Read P W and Larner J M 2007 Helical TomoTherapy in the treatment of central nervous system metastasis Neurosurg. Focus 22 1-6

Barra S et al 2015 Radiosurgery with helical TomoTherapy, outcomes for patients with one or multifocal brain metastasis Technol. Cancer Res. Treat. 14693-9

Bichay T J and Mayville A 2016 The continuous assessment of cranial motion in thermoplastic masks during cyberknife radiosurgery for trigeminal neuralgia Cureus 8 e607-e618

Boman E, Kapanen M, Laaksomaa M, Mäenpää H, Hyödynmaa S and Kellokumpu-Lehtinen P L 2016 Treatment accuracy without rotational setup corrections in intracranial SRT J. Appl. Clin. Med. Phys. 17 86-94 
Boswell S A, Jeraj R, Ruchala K J, Olivera G H, Jaradat H A, James J A, Gutierrez A, Pearson D, Frank G and Mackie T R 2005 A novel method to correct for pitch and yaw patient setup errors in helical TomoTherapy Med. Phys. 32 1630-9

Boswell S, Tomé W, Jeraj R, Jaradat H and Mackie T R 2006 Automatic registration of megavoltage to kilovoltage CT images in helical TomoTherapy: an evaluation of the setup verification process for the special case of a rigid head phantom Med. Phys. 33 4395-404

Cho Y H, Lee J M, Lee D, Park J H, Yoon K, Kim S O, Kwon D H, Kim J H, Kim C J and Roh S W 2015 Experiences on two different stereotactic radiosurgery modalities of gamma knife and cyberknife in treating brain metastases Acta Neurochir. 157 2003-9

Dieterich S et al 2011 Report of AAPM TG 135: quality assurance for robotic radiosurgery Med. Phys. 38 2914-36

Elson A, Walker A, Bovi J A and Schultz C 2015 Use of helical TomoTherapy for the focal hypofractionated treatment of limited brain metastases in the initial and recurrent setting Front Oncol. 5 1-6

Floriano A, Santa-Olalla I and Sanchez-Reyes A 2013 Initial evaluation of intrafraction motion using frameless CyberKnife VSI system Rep. Pract. Oncol. Radiother. 18 173-8

Frakes J M et al 2015 Potential role for linac-based stereotactic radiosurgery for the treatment of 5 or more radioresistant melanoma brain metastases J. Neurosurg. 123 1261-7

Gevaert T, Verellen D, Engels B, Depuydt T, Heuninckx K, Tournel K, Duchateau M, Reynders T and De Ridder M 2012 Clinical evaluation of a robotic 6-degree of freedom treatment couch for frameless radiosurgery Int. J. Radiat. Oncol. Biol. Phys. 83 467-74

Greto D, Pallotta S, Masi L, Talamonti C, Marrazzo L, Doro R, Saieva C, Scoccianti S, Desideri I and Livi L 2017 A dosimetric comparison between cyberknife and tomotherapy treatment plans for single brain metastasis Radiol. Med. 122 392-7

Han C, Liu A, Schultheiss T E, Pezner R D, Chen Y J and Wong JY 2006 Dosimetric comparisons of helical TomoTherapy treatment plans and step-and-shoot intensity-modulated radiosurgery treatment plans in intracranial stereotactic radiosurgery Int. J. Radiat. Oncol. Biol. Phys. 65 608-16

Kumar T, Rakowski J, Zhao B, Alkhafaji M, Burmeister J, Austin T and Vlachaki M 2010 Helical TomoTherapy versus stereotactic gamma knife radiosurgery in the treatment of single and multiple brain tumors: a dosimetric comparison J. Appl. Clin. Med. Phys. 11 27-41

Langen K M, Papanikolaou N, Balog J, Crilly R, Followill D, Goddu S M, Grant W, Olivera G, Ramsey C R and Shi C 2010 QA for helical TomoTherapy: report of the AAPM Task Group 148 Med. Phys. 37 4817-53

Lee A and Yamada Y J 2017 Stereotactic radiosurgery for multiple brain metastases J. Phys.: Conf. Ser. 777 1-5

Levegrün S, Pöttgen C, Wittig A, Lübcke W, Abu Jawad J and Stuschke M 2013 Helical TomoTherapy for whole-brain irradiation with integrated boost to multiple brain metastases: evaluation of dose distribution characteristics and comparison with alternative techniques Int. J. Radiat. Oncol. Biol. Phys. 86 734-42

Mahan S L, Ramsey C R, Scaperoth D D, Chase D J and Byrne T E 2005 Evaluation of image-guided helical TomoTherapy for the retreatment of spinal metastasis Int. J. Radiat. Oncol. Biol. Phys. 63 1576-83

Marrazzo L, Zani M, Pallotta S, Arilli C, Casati M, Compagnucci A, Talamonti C and Bucciolini M 2015 Gafchromic EBT3 films for patient specific IMRT QA using a multichannel approach Phys. Med. 8 1035-42

Meeks S L, Harmon J F, Langen K M, Willoughby T R, Wagner T H and Kupelian P A 2005 Performance characterization of megavoltage computed tomography imaging on a helical TomoTherapy unit Med. Phys. 32 2673-81

Mege J P, Wenzhao S, Veres A, Auzac G, Diallo I and Lefkopoulos D 2016 Evaluation of MVCT imaging dose levels during helical IGRT: comparison between ion chamber, TLD, and EBT3 films J. Appl. Clin. Med. Phys. 17 143-57

Minniti G, Clarke E, Lanzetta G, Osti M, Trasimeni G, Bozzao A, Romano A and Enrici R 2011 Stereotactic radiosurgery for brain metastases: analysis of outcome and risk of brain radionecrosis Radiat. Oncol. 6 1-9

Nagai A, Shibamoto Y, Yoshida M, Wakamatsu K and Kikuchi Y 2014 Treatment of single or multiple brain metastases by hypofractionated stereotactic radiotherapy using helical TomoTherapy Int. J. Mol. Sci. 15 6910-24

Park H S, Wang E H, Rutter C E, Corso C D, Chiang V L and Yu J B 2016 Changing practice patterns of gamma knife versus linear acceleratorbased stereotactic radiosurgery for brain metastases in the us J. Neurosurg. 124 1018-24

Ramakrishna N, Rosca F, Friesen S, Tezcanli E, Zygmanszki P and Hacker F 2010 A clinical comparison of patient setup and intra-fraction motion using frame-based radiosurgery versus a frameless image-guided radiosurgery system for intracranial lesions Radiother. Oncol. 95 109-15

Ruchala K, Olivera G and Kapatoes J 2002 Limited-data image registration for radiotherapy positioning and verification Int. J. Radiat. Oncol. Biol. Phys. 54 592-605

Schell M, Bova F J, Larson D, Leavitt D, Lutz W, Podgorsak E and Wu A 1995 AAPM Report No. 54 Stereotactic Radiosurgery (Alexandria, VA: American Association of Physicists in Medicine)

Shah A P, Langen K M, Ruchala K J, Cox A, Kupelian P A and Meeks S L 2008 Patient dose from megavoltage computed tomography imaging Int. J. Radiat. Oncol. Biol. Phys. 70 1579-87

Soisson E T, Hardcastle N and Tomé W A 2010 Quality assurance of an image guided intracranial stereotactic positioning system for radiosurgery treatment with helical TomoTherapy J. Neuro-Oncol. $98277-85$

Soisson E T, Tomé W A, Richards G M and Mehta M P 2006 Comparison of linac based fractionated stereotactic radiotherapy and tomotherapy treatment plans for skull-base tumors Radiother. Oncol. 78 313-21

Treuer H, Hoevels M, Luyken K, Visser-Vandewalle V, Wirths J, Kocher M and Ruge M 2014 Intracranial stereotactic radiosurgery with an adapted linear accelerator versus robotic radiosurgery Strahlenther. Onkol. 191 470-6

Vaandering A, Lee J A, Renard L and Grégoire V 2009 Evaluation of MVCT protocols for brain and head and neck tumor patients treated with helical TomoTherapy Radiother. Oncol. 93 50-6

Woodford C, Yartsev S and Dyk JV 2007 Optimization of megavoltage CT scan registration settings for brain cancer treatments on tomotherapy Phys. Med. Biol. 52 N185-93

Wowra B, Muacevic A and Tonn J C 2009 Quality of radiosurgery for single brain metastases with respect to treatment technology: a matched-pair analysis J. Neuro-Oncol. 94 69-77

Yartsev S, Kron T and Van Dyk J 2007 Tomotherapy as a tool in image-guided radiation therapy (IGRT): current clinical experience and outcomes Biomed. Imaging Interv. J. 3 e17-e25 